Case Report

\title{
Treatment of Homozygous Type II Antithrombin Heparin- Binding Site Deficiency in Pregnancy
}

\author{
Hilde Fiskvik (D), ${ }^{1}$ Anne F. Jacobsen, ${ }^{2,3}$ Nina Iversen, ${ }^{4}$ Carola E. Henriksson, ${ }^{3,5}$ \\ and Eva-Marie Jacobsen ${ }^{1}$ \\ ${ }^{1}$ Department of Hematology, Oslo University Hospital, Oslo, Norway \\ ${ }^{2}$ Department of Obstetrics and Gynecology, Oslo University Hospital, Oslo, Norway \\ ${ }^{3}$ Institute of Clinical Medicine, University of Oslo, Norway \\ ${ }^{4}$ Department of Medical Genetics, Oslo University Hospital, Oslo, Norway \\ ${ }^{5}$ Department of Medical Biochemistry, Oslo University Hospital, Oslo, Norway \\ Correspondence should be addressed to Hilde Fiskvik; hilfis@ous-hf.no
}

Received 20 April 2021; Accepted 14 August 2021; Published 3 September 2021

Academic Editor: John P. Geisler

Copyright (c) 2021 Hilde Fiskvik et al. This is an open access article distributed under the Creative Commons Attribution License, which permits unrestricted use, distribution, and reproduction in any medium, provided the original work is properly cited.

Pregnancy is associated with an increased risk of venous thromboembolism (VTE). Previous VTE and severe thrombophilia are important risk factors. Our case was a 36-year-old woman, gravida 6, para 0, with antithrombin (AT) deficiency caused by a homozygous mutation in the heparin-binding site (HBS). Her history included seven prior VTEs, three early and two late pregnancy losses. She was prophylactically treated with both human plasma-derived AT concentrate (hpATC) and low molecular weight heparin (LMWH), resulting in a successful $6^{\text {th }}$ pregnancy and a healthy live born baby. There is limited evidence and guidance on the management of AT deficiency in pregnancy. Dosing and monitoring of anticoagulants, alone or together with hpATC, must be based on individual risk assessment. The severity of clinical manifestations varies with the type of AT deficiency. Characterization of the AT mutation may aid in the decision-making process and optimize pregnancy outcomes.

\section{Introduction}

AT is a major inhibitor of thrombin (factor (F) IIa), FXa, and several other coagulation factors. Upon binding to heparin, the inactivation rate of AT enhances by 500-1000 [1].

The prevalence of hereditary AT deficiency is 0.02$0.2 \%$ in the general population [2] and $1-5 \%$ in patients with VTE [3]. AT deficiency can be quantitative (type I), with decreased AT activity and antigen, or qualitative (type II), with decreased AT activity and normal or slightly decreased antigen. Type II AT deficiency is classified into three subtypes based on the site of the causative mutation and the functional consequences, reactive site (RS), HBS, or pleiotropic effects. Heterozygous type II AT RS deficiency is reported to have the greatest potential for thrombosis, and heterozygous type II AT HBS deficiency the least. However, case publications and small studies of patients with homozy- gous HBS mutations imply that this subgroup is associated with an extreme high risk of thrombosis and pregnancy complications [4-10].

\section{Case Presentation}

A 36-year-old Serbian woman was referred to our hospital with a history of multiple VTEs and pregnancy losses.

At 17 years, she was diagnosed with a right iliac deep vein thrombosis shortly after starting with combined oral contraceptive pills. Investigation of thrombophilia showed reduced AT activity (32\%). There was no family history of AT deficiency or VTE. She was treated with warfarin for one year.

At 21 years, she had an extensive left leg thrombosis. Due to substantial symptoms not subsiding on unfractionated heparin (UFH), hpATC was added. She was discharged on warfarin. 
At 22 years, she was pregnant for the first time. LMWH was started with fraxiparine 3800 IU daily from gestational week (GW) 5. While on fraxiparine, she experienced an unexplained intrauterine fetal death (IUFD) in GW 23, and postpartum, she developed VTE. At 28 years, she was pregnant for the second time. LMWH with dalteparin $5000 \mathrm{IU} \times 1$ was immediately started, but in GW 9, she suffered a miscarriage. Pregnant for the third time at 29 years, dalteparin $5000 \mathrm{IU} \times 2$ was combined with hpATC 1500 IU twice weekly from GW 8. In GW 22, she experienced IUFD, and eight days postpartum, she was admitted with portal vein and superior mesenteric vein thrombosis. HpATC was administered for five days, warfarin was bridged with dalteparin, and she was discharged with an INR target of 2.5. One month later, she was readmitted with extensive thrombosis in the left arm and was treated with hpATC (2000 IU $\times 3$ /weekly). INR was 2.0 on admission, and warfarin was intensified to an INR target of 3.5.

At 30 years, she was pregnant for the $4^{\text {th }}$ time. While on dalteparin $5000 \mathrm{IU} \times 2$ and aspirin, she experienced another early pregnancy loss in GW 11, followed by a thrombosis in left vena saphena magna.

Two years later, she was pregnant for the $5^{\text {th }}$ time and visited our hospital for the first time. Her previous history was not well documented, AT activity was $38 \mathrm{IU} / \mathrm{dL}$ (measurement based on FIIa inhibition), and she was using dalteparin $5000 \mathrm{IU} \times 1$ and aspirin. She once again suffered a miscarriage in GW 11. One week later, she was readmitted and diagnosed with portal vein thrombosis.

At 34 years, she was pregnant for the $6^{\text {th }}$ time and was switched from warfarin to dalteparin $100 \mathrm{IU} / \mathrm{kg} \times 2 /$ day $(7500 \mathrm{IU} \times 2)$ in GW 5. She was diagnosed with a homozygous mutation in exon 2, LEU131Phe, i.e., a type II AT HBS deficiency. HpATC was administered 2000 IU twice weekly. Due to consistently low AT activity around $40 \mathrm{IU} / \mathrm{dL}$, the dose was increased to $2000 \mathrm{IU}$ three times a week from GW 21 and further increased to $2500 \mathrm{IU} \times$ 3/weekly from GW 33 (equivalent to $32 \mathrm{IU} / \mathrm{kg}$ ). LMWH with dalteparin was also increased to $130 \mathrm{IU} / \mathrm{kg} \times 2 /$ day $(10000 \mathrm{IU} \times 2)$ in the third trimester.

She was admitted at week $36+1$ due to contractions. She was not in active labour, and a caesarean section (CS) was scheduled the following morning. The day before the CS, hpATC $3000 \mathrm{IU} \times 1$ was administered and continued for five days. The target AT activity was $80 \mathrm{IU} / \mathrm{dL}$ but reached only $64-78 \mathrm{IU} / \mathrm{dL}$ in the days around delivery. A healthy baby was delivered, and warfarin was restarted the day after CS with dalteparin bridging. Four days after delivery, the dose of hpATC was reduced from $3000 \mathrm{IU}$ daily to $2000 \mathrm{IU} \times 3$ weekly. Prophylactically, treatment with hpATC continued for 3 weeks postpartum due to her previous history of postpartum VTEs.

\section{Discussion}

Homozygous AT deficiency is not considered compatible with life except for homozygous HBS mutations which are characterized by arterial and venous thrombosis at young age and pregnancy complications [5-8, 10-12]. Our patient had seven VTEs and six pregnancies, before she succeeded, confirming the very high risk.

There are no randomized trials or guidelines on how to manage patients with HBS deficiency in pregnancy, leaving strategies largely based on case reports and expert opinions. Some authors have published guidance based on personal and family history of VTE $(15,21,23)$. We argue that the characterization of the subclass of AT deficiency, type, and site of mutation must be considered when deciding prophylaxis and treatment in pregnancy.

The most common indications for the use of AT concentrate in high-risk pregnant AT deficient women are management of VTE that occurs despite treatment with anticoagulants and prophylactic use in the peripartum period.

AT activity of $80-120 \mathrm{IU} / \mathrm{dL}$ has been recommended as target during therapeutic substitution with hpATC [13], but for prophylactic use in pregnancy, there are no guidelines on target AT activity.

In most reports on successful pregnancies in homozygous $\mathrm{HBS}$ patients $[6,8,10,14]$, patients received both hpATC and anticoagulants.

The obstetric history of our patient made her pregnancy high risk, both for VTE and pregnancy loss, and prompted the use of both hpATC and LMWH. Our patient first received $32 \mathrm{IU} / \mathrm{kg}$ twice a week and then three times weekly, and the frequency was based on the biological half-life of hpATC which is about three days. Considering the lack of recommendations for target AT activity for prophylaxis, we chose to substitute with approximately $30 \mathrm{IU} / \mathrm{kg}$. The highest AT activity was achieved in the days around delivery, where AT activity was 64-78 IU/dL.

The anticoagulant effect of LMWH is highly dependent on AT, and low levels of functional AT may lead to heparin resistance and reduced effect of LMWH [3]. It is thought that concomitant administering of hpATC to heparin will overcome the heparin resistance. Routine measurements of anti-FXa activity to guide dosing of LMWH in healthy pregnancy are not recommended [15], and evidence-based guidance to appropriate peak and through levels is limited. However, in high-risk women with AT deficiency, Bramham et al. [16] have suggested monitoring of LMWH with target peak levels of 0,5-1 kIU/L and through levels $>0,1 \mathrm{kIU} / \mathrm{L}$. In spite of LMWH $260 \mathrm{IU} / \mathrm{kg} /$ day and hpATC $32 \mathrm{IU} / \mathrm{kg}$ three times weekly, anti-FXa activity in our patient only varied between 0,1 and $0,2 \mathrm{kIU} / \mathrm{L}$ (liquid anti-Xa, one-stage chromogenic assay with no exogenous AT). Noteworthy, in a few previous cases of AT deficiency where the aim was to dose-adjust LMWH based on anti-FXa measurements [17, 18], anti-FXa activity was still low despite concurrent administration of hpATC. When VTE occurs after the first trimester despite LMWH 200 IU/day, an alternative to adding hpATC may be to switch to warfarin and return to hpATC and LMWH around delivery. This treatment option has been described successfully in case reports $[17,19,20]$. In the postpartum setting, a rapid transition to a nonheparin anticoagulant is recommended to avoid the problem of heparin resistance. In our patient, with one exception, the VTEs occurred on LMWH. 


\section{Conclusion}

AT deficiency is a high-risk thrombophilia, but the risk of VTE in pregnancy varies significantly between the different subclasses. Decisions concerning prophylactic and therapeutic treatment and monitoring of treatment in pregnancy may be facilitated if the type and site of the mutation are known. There is a need for recommendations for both prophylaxis and therapeutic treatment that takes into account the genetic profile, personal and family history of VTE, and obstetric history in each individual patient.

\section{Conflicts of Interest}

The authors have no conflicts of interest to disclose.

\section{Authors' Contributions}

All authors contributed equally to design the study and critically evaluated and discussed the ongoing clinical issues or analyses. Hilde Fiskvik drafted the manuscript.

\section{References}

[1] D. Hernandez-Espinosa, A. Ordonez, V. Vicente, and J. Corral, "Factors with conformational effects on haemostatic serpins: implications in thrombosis," Thrombosis and Haemostasis, vol. 98, no. 9, pp. 557-563, 2007.

[2] R. C. Tait, I. D. Walker, D. J. Perry et al., "Prevalence of antithrombin deficiency in the healthy population," British Journal of Haematology, vol. 87, no. 1, pp. 106-112, 1994.

[3] M. M. Patnaik and S. Moll, "Inherited antithrombin deficiency: a review," Haemophilia, vol. 14, no. 6, pp. 1229-1239, 2008.

[4] G. Finazzi, R. Caccia, and T. Barbui, "Different prevalence of thromboembolism in the subtypes of congenital antithrombin III deficiency: review of 404 cases," Thrombosis and Haemostasis, vol. 58, no. 4, p. 1094, 1987.

[5] R. Gindele, Z. Oláh, P. Ilonczai et al., "Founder effect is responsible for the p.Leu131Phe heparin-binding-site antithrombin mutation common in Hungary: phenotype analysis in a large cohort," Journal of Thrombosis and Haemostasis, vol. 14, no. 4, pp. 704-715, 2016.

[6] P. Ilonczai, Z. Oláh, A. Selmeczi et al., "Management and outcome of pregnancies in women with antithrombin deficiency: a single-center experience and review of literature," Blood Coagulation \& Fibrinolysis, vol. 26, no. 7, pp. 798-804, 2015.

[7] C. Orlando, O. Heylen, W. Lissens, and K. Jochmans, "Antithrombin heparin binding site deficiency: a challenging diagnosis of a not so benign thrombophilia," Thrombosis Research, vol. 135, no. 6, pp. 1179-1185, 2015.

[8] J. Kraft, R. Sunder-Plassmann, C. Mannhalter et al., "Women with homozygous AT deficiency type II heparin-binding site (HBS) are at high risk of pregnancy loss and pregnancy complications," Annals of Hematology, vol. 96, no. 6, pp. 10231031, 2017.

[9] B. Luxembourg, A. Pavlova, C. Geisen et al., "Impact of the type of SERPINC1 mutation and subtype of antithrombin deficiency on the thrombotic phenotype in hereditary antithrombin deficiency," Thrombosis and Haemostasis, vol. 111, no. 2, pp. 249-257, 2014.
[10] M. Kovac, G. Mitic, Z. Mikovic et al., "The influence of specific mutations in the AT gene (SERPINC1) on the type of pregnancy related complications," Thrombosis Research, vol. 173, pp. 12-19, 2019.

[11] B. Pearson-Stuttard, C. Bagot, E. Ciantar et al., "Severe antithrombin deficiency in pregnancy: achieving adequate anticoagulation," Obstetric medicine, vol. 12, no. 1, pp. 45-51, 2019.

[12] M. Kovac, G. Mitic, I. Djilas et al., "Genotype phenotype correlation in a pediatric population with antithrombin deficiency," European Journal of Pediatrics, vol. 178, no. 10, pp. 1471-1478, 2019.

[13] M. Refaei, L. Xing, W. Lim, M. Crowther, and K. Boonyawat, "Management of venous thromboembolism in patients with hereditary antithrombin deficiency and pregnancy: case report and review of the literature," Case reports in hematology, vol. 2017, Article ID 9261351, 11 pages, 2017.

[14] M. Kovac, G. Mitic, P. Miljic et al., "Poor pregnancy outcome in women with homozygous type-II HBS antithrombin deficiency," Thrombosis Research, vol. 133, no. 6, pp. 1158-1160, 2014.

[15] S. M. Bates, A. Rajasekhar, S. Middeldorp et al., "American Society of Hematology 2018 guidelines for management of venous thromboembolism: venous thromboembolism in the context of pregnancy," Blood Advances, vol. 2, no. 22, pp. 3317-3359, 2018.

[16] K. Bramham, A. Retter, S. E. Robinson, M. Mitchell, G. W. Moore, and B. J. Hunt, "How I treat heterozygous hereditary antithrombin deficiency in pregnancy," Thrombosis and Haemostasis, vol. 110, no. 9, pp. 550-559, 2013.

[17] C. Pascual, C. Muñoz, A. R. Huerta et al., "A new case of successful outcome of pregnancy in a carrier of homozygous type II (L99F) antithrombin deficiency," Blood Coagulation \& Fibrinolysis, vol. 25, no. 1, pp. 74-76, 2014.

[18] P. Tsikouras, A. Christoforidou, A. Bothou et al., "Overcoming heparin resistance in pregnant women with antithrombin deficiency: a case report and review of the literature," Journal of Medical Case Reports, vol. 12, no. 1, p. 169, 2018.

[19] G. Alguel, K. Jochmans, R. Simanek et al., "Successful outcome in a pregnant woman with homozygous antithrombin deficiency," Thrombosis and Haemostasis, vol. 98, no. 12, pp. 1377-1378, 2007.

[20] A. García-Botella, S. Asenjo, M. E. de la Morena-Barrio et al., "First case with antithrombin deficiency, mesenteric vein thrombosis and pregnancy: multidisciplinary diagnosis and successful management," Thrombosis Research, vol. 144, pp. 72-75, 2016. 